\title{
ON QUASI-PSEUDOCONFORMALITY IN SEVERAL COMPLEX VARIABLES ${ }^{1}$ )
}

\author{
BY \\ RICARDO NIRENBERG
}

1. Introduction. If $\zeta=f(z)$ is an orientation preserving diffeomorphism of a plane domain $D$ on a domain $E$, its constant of quasiconformality $K[f]$ is defined as follows: consider the jacobian matrix $T_{p}$ of the mapping $f$ at the point $p \in D$. Denote by $J$ the matrix of the transformation $z \rightarrow(-1)^{1 / 2} z$, and consider the commutator $\hat{T}_{p}=T_{p} J T_{p}^{-1} J^{-1} . \hat{T}_{p}$ is a positive definite symmetric matrix with determinant 1 : its eigenvalues are $K\left[T_{p}\right]$ and $\left(K\left[T_{p}\right]\right)^{-1}$, where $K\left[T_{p}\right] \geqq 1$, and $K\left[T_{p}\right]=1$ if and only if $T$ commutes with $J$, that is, if and only if $T$ is an analytic matrix, representing the transformation of the plane given by multiplication by a complex number. We then set

$$
K[f]=\sup \left\{K\left[T_{p}\right] ; p \in D\right\} .
$$

$K\left[T_{p}\right]$ can be equivalently defined in the usual way as the ratio of the axes of the "infinitesimal ellipse" into which an "infinitesimal circle" around $p$ is mapped. That these two definitions coincide refiects the fact that in $\boldsymbol{C}^{1}$ analyticity and conformality are one and the same notion. Of course, this is no longer the case for more than one complex variable. One can define quasiconformal transformations of domains in $R^{n}$, for $n>2$, by considering ratios of the axes of the ellipsoid into which an infinitesimal sphere is mapped, as F. W. Gehring and others have done [4], but this is in no way related to the analytic structure of a domain in $\boldsymbol{C}^{n}$, for $n>1$. On the other hand, for $n>1$, the commutator of a $2 n \times 2 n$ matrix $T$ with the matrix of the transformation $z_{i} \rightarrow(-1)^{1 / 2} z_{i}, i=1, \ldots, n$, is no longer symmetric, not even diagonalizable, so that we cannot generalize our first definition of $K[T]$ to several complex variables.

To make the problem of generalizing quasiconformality to several complex variables precise, in $\$ 2$ we give a set of axioms defining the notion of a "constant of quasi-pseudoconformality." They can be summed up as follows: we are given a nonempty category $\mathscr{E}$ of domains in $C^{n}$ whose morphisms are called "quasipseudoconformal maps." The category $\mathscr{E}$ contains all the domains analytically equivalent to a domain in $\mathscr{E}$, and pseudoconformal (i.e., bi-analytic) maps are always morphisms. To each morphism there is associated a real number $K \geqq 1$, which is 1 for pseudoconformal transformations, and which remains invariant

Received by the editors June 6, 1966.

(1) This research was carried out with the support of a Pre-Doctoral Fellowship at the Courant Institute of Mathematical Sciences, New York University. 
under right or left composition with pseudoconformal maps. Furthermore, $K[\mathscr{T} \circ \mathscr{S}]$ is less than or equal to some bounded function of $K[\mathscr{T}]$ and $K[\mathscr{S}]$. All these properties are essential in the theory of quasiconformal maps in the plane. Then we give two more axioms, as little restrictive as seems possible, to exclude trivial situations and to make our "constant of quasi-pseudoconformality" bear some relation to the one already defined for domains in the complex plane.

But the situation in $C^{n}, n>1$, as it might be expected, is essentially different from that in $C^{1}$, and thus in $\$ 3$ we state two theorems of a negative character. From the second one we get the following corollary: if the category $\mathscr{E}$ is large enough (if, for example, $\mathscr{E}$ contains all domains included in a domain belonging to $\mathscr{E}$ ), then the constant of quasi-pseudoconformality cannot be, as in the plane, of a local character. That is, $K[\mathscr{T}]$ cannot be of the form $\sup K_{P}[\mathscr{T}]$, where $K_{P}[\mathscr{T}]$ depends on the behavior of $\mathscr{T}$ near the point $P$, but not on the particular domain on which $\mathscr{T}$ is defined.

St. Bergman introduced in [2] (see also [5]) a class of quasi-pseudoconformal transformations and a constant of quasi-pseudoconformality of a local type, but with his definition $K[\mathscr{T} \circ \mathscr{S}]$ is not bounded in terms of $K[\mathscr{T}]$ and $K[\mathscr{S}]$ and besides, any transformation of the form:

$$
Z_{1}=f_{1}\left(z_{1}, z_{2}\right), \quad Z_{2}=f_{2}\left(z_{2}\right)
$$

with $f_{1}$ analytic in $z_{1}$ but only differentiable with respect to $z_{2}$, and $f_{2}$ analytic, has a constant equal to 1 , the same as analytic transformations. For another class of quasi-pseudoconformal maps in a special category of subdomains of $\boldsymbol{C}^{2}$ (Reinhardt circular domains), see [3] and [5].

I am greatly indebted to Professor Lipman Bers, who directed the doctoral dissertation of which the present work is a part.

2. The definition of quasi-pseudoconformality. Suppose we are given a nonempty family $\mathscr{E}$ of domains in $C^{n}$ with the property that if $D \in \mathscr{E}$ and there is a bi-analytic map $\mathscr{A}$ of $D$ onto a domain $E$ (that is, $D$ and $E$ are analytically equivalent), then $E \in \mathscr{E}$. Also, we will assume that $\Delta_{n}(0,1)$, the unit polydisk in $C^{n}$, belongs to $\mathscr{E}$. Further, suppose that for every pair of domains $D, E$ in $\mathscr{E}$, we have a family of homeomorphisms $\mathrm{QPC}(D, E) \subset \operatorname{Hom}(D, E)$ (which may sometimes be empty), satisfying the following conditions:

(QPC-1) If $\mathscr{T} \in \mathrm{QPC}\left(D^{\prime}, D\right)$ and $\mathscr{S} \in \mathrm{QPC}(D, E)$, then $\mathscr{S} \circ \mathscr{T} \in \mathrm{QPC}\left(D^{\prime}, E\right)$.

(QPC-2) If $\mathscr{T} \in \mathrm{QPC}(D, E)$, then $\mathscr{T}^{-1} \in \mathrm{QPC}(E, D)$.

(QPC-3) $\quad \mathrm{QPC}(D, E) \supset \mathscr{A}(D, E)$, the bi-analytic maps of $D$ onto $E$.

In particular, if $D=E, \mathrm{QPC}(D, E)$ will be written $\mathrm{QPC}(D)$. Next, for every pair of domains $D, E \in \mathscr{E}$ there is a "constant of quasi-pseudoconformality": a function 
$K(D, E)$ defined on $\mathrm{QPC}(D, E)$, whose values are real and $\geqq 1$, satisfying the following requirements:

$$
\begin{aligned}
& \text { If } \mathscr{T} \in \mathscr{A}(D, E) \text {, then } K(D, E)[\mathscr{T}]=1 \text {. } \\
& \text { If } \mathscr{S}_{1} \in \mathscr{A}\left(D, D^{\prime}\right), \mathscr{T} \in \mathrm{QPC}\left(D^{\prime}, E^{\prime}\right) \text { and } \mathscr{S}_{2} \in \mathscr{A}\left(E^{\prime}, E\right) \text {, } \\
& \text { then } K(D, E)\left[\mathscr{S}_{2} \circ \mathscr{T} \circ \mathscr{S}_{1}\right]=K\left(D^{\prime}, E^{\prime}\right)[\mathscr{T}] \\
& \text { If } \mathscr{T}_{1} \in \mathrm{QPC}(D, E) \text { and } \mathscr{T}_{2} \in \mathrm{QPC}(E, F) \text {, then } \\
& K(D, F)\left[\mathscr{T}_{2} \circ \mathscr{T}_{1}\right] \leqq K(E, F)\left[\mathscr{T}_{2}\right] K(D, E)\left[\mathscr{T}_{1}\right] \text {. }
\end{aligned}
$$

This last requirement can be weakened by asking only that if $K(D, E)\left[\mathscr{T}_{1}\right]$ and $K(E, F)\left[\mathscr{T}_{2}\right]$ are uniformly bounded for $\mathscr{T}_{1}$ and $\mathscr{T}_{2}$ in some set of transformations, $K(D, F)\left[\mathscr{T}_{2} \circ \mathscr{T}_{1}\right]$ should be bounded.

There are trivial ways of defining the families $\mathscr{E}, \mathrm{QPC}$, and the functions $K$ fulfilling our conditions. For example:

(i) For any pair of domains $D, E$ in $C^{n}$ set $\mathrm{QPC}(D, E)=\mathscr{A}(D, E)$, and $K=1$ identically.

(ii) For any pair of domains set $\operatorname{QPC}(D, E)=\operatorname{Hom}(D, E)$, and $K[\mathscr{T}]=1$ if $\mathscr{T} \in \mathscr{A}(D, E) ; K[\mathscr{T}]=K_{0}$, say, otherwise, where $K_{0}$ is a real number $\geqq 1$.

Evidently this is not what we are looking for, so if we want to consider a reasonable generalization of the notion of quasiconformality in one complex variable, we should add some more axioms. A possible set of additional axioms that seems quite natural and as little restrictive as possible, is the following:

(QPC-4) $B \subset C^{1}$, and if $T$ is a linear self-mapping of $B$, then

$$
\mathscr{T}=T \oplus \cdots \oplus T \in \mathrm{QPC}(D) \text {. }
$$

This axiom excludes our example (i), but not (ii). Then we require $K$ to satisfy:

If again $D$ is a product domain, and a coordinate plane, that is, a set obtained by fixing $n-1$ of the complex coordinates, is mapped into itself

(K-4) by a family of mappings $\mathscr{T}_{i} \in \mathrm{QPC}(D)$ and $K(D)\left[\mathscr{T}_{i}\right]$ is uniformly bounded, then the $\mathscr{T}_{i}$ 's restricted to this plane have a constant of quasiconformality which is also bounded.

This last axiom excludes example (ii); it means that there are not too many quasipseudoconformal maps; axiom (QPC-4) says, of course, that there are not too few of them.

3. Two examples and two negative results. There is a simple, albeit uninteresting situation in which all our axioms are satisfied. Take $\Delta_{n}(0,1)$, the unit polydisk in $\boldsymbol{C}^{n}$, and consider the family $\mathscr{E}$ of all domains in $\boldsymbol{C}^{n}$ analytically equivalent to $\Delta_{n}(0,1)$. For QPC $\left(\Delta_{n}(0,1)\right)$ take the group of mappings of the form

$$
\zeta_{i}=\phi_{i}\left(z_{\sigma(i)}\right), \quad i=1,2, \ldots, n,
$$


where the $\phi_{i}$ 's are quasiconformal maps of the unit disk onto itself, and $\sigma(i)$ is a permutation of the numbers $(1,2, \ldots, n)$. It is a well-known fact that all analytic automorphisms of the polydisk are of the form (1), with $\phi_{i}$ an analytic automorphism of the disk for $i=1,2, \ldots, n$ (cf. [1]). Hence QPC $\left(\Delta_{n}(0,1)\right) \supset \mathscr{A}\left(\Delta_{n}(0,1)\right)$ as required. If the domains $D, E$ are equivalent to the polydisk, $\operatorname{QPC}(D, E)$ will consist of those homeomorphisms $\mathscr{T}: D \rightarrow E$ such that $\mathscr{S}_{2} \circ \mathscr{T} \circ \mathscr{S}_{1} \in \mathrm{QPC}\left(\Delta_{n}(0,1)\right)$, where $\mathscr{S}_{1}$ and $\mathscr{S}_{2}$ are bi-analytic maps of $\Delta_{n}(0,1)$ onto $D$ and $E$ respectively. It is easy to see that the fact that $\mathscr{S}_{2} \circ \mathscr{T} \circ \mathscr{S}_{1}$ belongs to $\mathrm{QPC}\left(\Delta_{n}(0,1)\right)$ is independent of the choice of $\mathscr{S}_{1}$ and $\mathscr{S}_{2}$. For $\mathscr{T} \in \mathrm{QPC}\left(\Delta_{n}(0,1)\right)$ we define

$$
K\left(\Delta_{n}(0,1)\right)[\mathscr{T}]=\max \left\{K\left[\phi_{i}\right] ; i=1, \ldots, n\right\},
$$

where $K\left[\phi_{i}\right]$ is the constant of quasiconformality in the plane. It is then obvious how to define $K(D, E)$ for $D, E \in \mathscr{E}$, and equally obvious how to verify that all our axioms are satisfied. Of course, this setup gives us nothing new: our family $\mathscr{E}$ is too small (the smallest possible under our conditions), and in it, the theory of quasi-pseudoconformal maps is reduced to the theory in $C^{\mathbf{1}}$. Thus, we would like to consider larger families of domains; the larger, the better.

But the situation in $C^{n}$ is essentially different from that in $C^{1}$, as we see from the following

THEOREM 1. If $\mathscr{E}, \mathrm{QPC}$ and $K$ satisfy all axioms (QPC-1)-(QPC-4) and (K-1)$(\mathrm{K}-4)$, and if $n>1, C^{n}$ cannot belong to $\mathscr{E}$.

Before proving this theorem we observe that $\mathscr{E}$ can contain all bounded domains. In fact, take $\mathscr{E}$ to be the family of bounded domains of $C^{n}$ (plus those equivalent to a bounded domain); in each of these domains consider an invariant metric (for example, the Carathéodory or the Bergman metric [1]), and take as $\mathrm{QPC}(D, E)$ the transformations that are quasi-isometries between the metric spaces $D$ and $E$. For such a transformation $\mathscr{T}$ there exists a constant $K$ such that

$$
K^{-1} d_{D}(P, Q) \leqq d_{E}(\mathscr{T}(P), \mathscr{T}(Q)) \leqq K d_{D}(P, Q)
$$

for every pair of points $P, Q$ in $D$, where $d_{D}$ and $d_{E}$ are the metrics on $D$ and $E$ respectively. We then define $K(D, E)[\mathscr{T}]$ as the smallest possible constant for which (2) is true. Properties (QPC-1)-(QPC-3) and (K-1)-(K-3) are quite easy to verify. Property $(\mathrm{K}-4)$ is proved by noting first that the metric induced on a coordinate plane by the invariant distance in $D$ is, save for normalization, the noneuclidean Poincaré metric, and secondly, that a quasi-isometry of the Poincaré metric is always a quasiconformal map whose constant of quasiconformality is bounded in terms of the constant $K$ in (2). As for axiom (QPC-4), the following stronger statement is actually true: if $f_{i}: B \rightarrow B, i=1, \ldots, n$, are quasi-isometries of the Poincaré metric, then

$$
f_{1} \oplus \cdots \oplus f_{n} \in \mathrm{QPC}(B \times \cdots \times B) .
$$


Naturally, in a good generalization of quasiconformality (3) should be true whenever the $f_{i}$ 's are just quasiconformal automorphisms of $\boldsymbol{B}$ (which are strictly more than quasi-isometries of the Poincare metric), but the author was not able to construct a setup in which this happens, with $\mathscr{E}$ large enough so as to contain all bounded, geometrically convex domains. In any case, for such a category $\mathscr{E}$ the constant of quasi-pseudoconformality cannot be a local one, as it turns out readily from the following theorem.

THEOREM 2. If $n>1$, there is no definition of quasi-pseudoconformality satisfying, in addition to (QPC-1), .., (QPC-4), (K-1), .., (K-4), the following axioms:

(i) There is a bounded domain $E \in \mathscr{E}$ such that all its real-linear images are in $\mathscr{E}$.

(ii) If $D, E, D^{\prime}, E^{\prime}$ belong to $\mathscr{E}, D^{\prime} \subset D, E^{\prime} \subset E$, and $\mathscr{T} \in \mathrm{QPC}(D, E)$, then if $D^{\prime}$ is sent by $\mathscr{T}$ onto $E^{\prime}, \mathscr{T} \in \mathrm{QPC}\left(D^{\prime}, E^{\prime}\right)$ and furthermore

$$
K\left(D^{\prime}, E^{\prime}\right)\left[\mathscr{T} \mid D^{\prime}\right] \leqq K(D, E)[\mathscr{T}] .
$$

REMARK. Note that our last example does not satisfy property (ii).

4. Proof of Theorems 1 and 2. For the proof of Theorem 1 it is convenient to begin with some elementary remarks on linear algebra. Without loss of generality we will consider the case $n=2$.

(a) If $D \subset C^{2}$ is invariant under $J$ (multiplication by $(-1)^{1 / 2}$ of both variables), then if the matrix $T$ belongs to $\mathrm{QPC}(D), \hat{T}$ also belongs to $\mathrm{QPC}(D)$, and if the matrices $S$ and $T$ are such that $\hat{S}=B \hat{T} B^{-1}$ where $B$ is analytic, then there exists an analytic matrix $A$ such that $S=B T A$, and therefore $K(D)[S]=K(D)[T]$.

(b) LEMMA. The matrix $\hat{T}$ can be carried to a canonical form by a similarity with an analytic matrix (if $\hat{T}$ has complex eigenvalues, the analytic matrix is a complex 4 by 4 matrix commuting with $J$ ). If $\hat{T}$ is diagonalizable, the canonical form is the diagonal one.

Proof. If $\lambda$ is an eigenvalue of $\hat{T}$ and $X$ an eigenvector for $\lambda$, then $\lambda^{-1}$ is also an eigenvalue, and $J X$ is an eigenvector for it. Then by choosing a new basis of the form $\{X, J X, Y, J Y\}$, where $X$ is an eigenvector of $\hat{T}$ and $Y$ is any vector not in the subspace $[X, J X]$ spanned by $X$ and $J X$, such that $\hat{T} Y$ lies in the subspace $[X, J X, Y]$, we can reduce $\hat{T}$ to triangular form. Observe that the matrix $B$ corresponding to this change of basis is analytic. If the matrix $\hat{T}$ is diagonalizable, $B \hat{T} B^{-1}$ is diagonal. Now, if $\hat{T}$ is not diagonalizable, its eigenvalues are $\lambda, \lambda^{-1}$, $\lambda, \lambda^{-1}$. We have:

$$
\hat{T} Y=a X+b J X+\lambda Y .
$$

But then, applying $J$ on both sides:

$$
\left(J T J^{-1} T^{-1}\right)(J Y)=a J X-b X+\lambda J Y,
$$

hence applying $\hat{T}$ on both sides we get

$$
J Y=a \hat{T} J X-b \hat{T} X+\lambda \hat{T} J Y=a \lambda^{-1} J X-b J X+\lambda \hat{T} J Y .
$$


So,

$$
\hat{T}(J Y)=b X-a \lambda^{-2} J X+\lambda^{-1} J Y,
$$

and we have proved that $\hat{T}$ is carried to the following canonical form:

(4)

$$
\left[\begin{array}{llll}
\lambda & 0 & a & b \\
0 & \lambda^{-1} & b & -a \lambda^{-2} \\
0 & 0 & \lambda & 0 \\
0 & 0 & 0 & \lambda^{-1}
\end{array}\right] . \quad \text { Q.E.D. }
$$

(c) Conversely, for any $\lambda \neq 0, a, b$, there is a matrix $T$ such that $\hat{T}$ has the form (4). Take, for example,

$$
\left[\begin{array}{cccc}
\lambda & 0 & 0 & 0 \\
0 & 1 & b & -a \lambda^{-1} \\
0 & 0 & \lambda & 0 \\
0 & 0 & 0 & 1
\end{array}\right]
$$

Proof of Theorem 1. By axiom (QPC-4), any matrix of the form:

$$
U=\left[\begin{array}{llll}
\lambda & 0 & 0 & 0 \\
0 & 1 & 0 & 0 \\
0 & 0 & \lambda & 0 \\
0 & 0 & 0 & 1
\end{array}\right], \quad \lambda \neq 0,
$$

is in $\mathrm{QPC}\left(C^{2}\right)$. If we consider the matrix

$$
S=\left[\begin{array}{rrrr}
\lambda & 0 & 0 & -\lambda \alpha \\
0 & 1 & \alpha & 0 \\
0 & 0 & \lambda & 0 \\
0 & 0 & 0 & 1
\end{array}\right]
$$

we see that $S=0$ for any $\alpha$, so $S$ is also in $\operatorname{QPC}\left(C^{2}\right)$ by remark (a). Then the matrix $S^{2}$ belongs to $\mathrm{QPC}\left(C^{2}\right)$, by (QPC-1). But

$$
\hat{S^{2}}=\left[\begin{array}{llll}
\lambda^{2} & 0 & 0 & \beta \\
0 & \lambda^{-2} & \beta & 0 \\
0 & 0 & \lambda^{2} & 0 \\
0 & 0 & 0 & \lambda^{-2}
\end{array}\right]
$$

where $\beta=\alpha(\lambda+1)\left(1-\lambda^{-1}\right)$. Now if

$$
T_{1}=\left[\begin{array}{llll}
\lambda^{2} & 0 & 0 & 0 \\
0 & 1 & \beta & 0 \\
0 & 0 & \lambda^{2} & 0 \\
0 & 0 & 0 & 1
\end{array}\right],
$$


then $\hat{T}_{1}=\hat{S}^{2}$ by remark (c), and therefore $T_{1} \in \mathrm{QPC}\left(C^{2}\right)$. Finally, the matrix

$$
T=\left[\begin{array}{llll}
\lambda^{-2} & 0 & 0 & 0 \\
0 & 1 & 0 & 0 \\
0 & 0 & \lambda^{-2} & 0 \\
0 & 0 & 0 & 1
\end{array}\right] \quad T_{1}=\left[\begin{array}{llll}
1 & 0 & 0 & 0 \\
0 & 1 & \beta & 0 \\
0 & 0 & 1 & 0 \\
0 & 0 & 0 & 1
\end{array}\right]
$$

is in $\mathrm{QPC}\left(\boldsymbol{C}^{2}\right)$, by $(\mathrm{QPC}-1)$ and (QPC-4). Therefore for any real number $a$, the matrix

$$
T_{a}=\left[\begin{array}{llll}
1 & 0 & 0 & 0 \\
0 & 1 & a & 0 \\
0 & 0 & 1 & 0 \\
0 & 0 & 0 & 1
\end{array}\right]
$$

belongs to QPC $\left(C^{2}\right) . K\left(C^{2}\right)\left[T_{a}\right]$ does not depend on $a$ when $a \neq 0$, because as it is easy to verify, all the matrices $T_{a}, a \neq 0$, are analytically similar. Now consider the matrix

$$
\Omega_{(a, b)}=T_{a} A T_{b} \in \mathrm{QPC}\left(C^{2}\right)
$$

for $a$ and $b$ different from zero, and where the analytic matrix $A$ is interchanging of the variables $z_{1}$ and $z_{2}$. One finds that

$$
\Omega_{(a, b)}=\left[\begin{array}{llll}
1 & 0 & 0 & a \\
0 & a b+1 & a & 0 \\
0 & b & 1 & 0 \\
b & 0 & 0 & a b+1
\end{array}\right]
$$

and that the minimal polynomial of $\Omega_{(a, b)}$ is $x^{2}-(2+a b) x+1$ if $a b \neq-4$. In this case the roots are different and therefore $\Omega_{(a, b)}$ is diagonalizable. Thus, by an analytic similarity, it can be carried to the form

$$
M_{\mu}=\left[\begin{array}{llll}
\mu & 0 & 0 & 0 \\
0 & \mu^{-1} & 0 & 0 \\
0 & 0 & \mu & 0 \\
0 & 0 & 0 & \mu^{-1}
\end{array}\right]
$$

where $\mu+\mu^{-1}=2+a b$. Hence, for some analytic matrices $A$ and $B, A \Omega_{(a, b)} B$ is equal to the matrix $M_{\mu}$. But, for any $a, b \neq 0$, by (K-3):

$$
\begin{aligned}
K\left(C^{2}\right)\left[A \Omega_{(a, b)} B\right] & =K\left(C^{2}\right)\left[\Omega_{(a, b)}\right] \leqq K\left(C^{2}\right)\left[T_{a}\right] \cdot K\left(C^{2}\right)\left[T_{b}\right] \\
& =\left(K\left(C^{2}\right)[T]\right)^{2} .
\end{aligned}
$$

So, for any $\mu$ the constant of quasi-pseudoconformality of the matrix $M_{\mu}$ is uniformly bounded. But this contradicts axiom (K-4), for the matrix $M_{\mu}$ restricted to the plane $z_{2}=0$, say, has an arbitrarily large constant of quasiconformality. Q.E.D. 
Proof of Theorem 2. Again, without loss of generality, we assume $\boldsymbol{n}$ to be 2 . Consider the product domain $D=B \times B$, where $B=\{z ; \operatorname{Im} z>0\} \subset C$. Then the matrix $U$ at the beginning of the proof of Theorem 1 belongs, by (QPC-4), to $\mathrm{QPC}(D)$. We can assume that the bounded domain $E$ is included in $D$, so by (ii), $U \in \mathrm{QPC}(E, U(E))$. Now, from (QPC-1), (QPC-3), (K-2) and (ii) we have that if a linear map $T$ is in QPC $(V, T(V))$ for a bounded domain $V \in \mathscr{E}$, then $T$ is also in QPC $\left(V^{\prime}, T\left(V^{\prime}\right)\right)$ for any bounded domain $V^{\prime} \in \mathscr{E}$ (because scalar matrices and translations are analytic transformations), and that $K\left(V^{\prime}, T\left(V^{\prime}\right)\right)[T]$ does not depend on the bounded domain $V^{\prime} \in \mathscr{E}$. We can then repeat the construction in the proof of Theorem 1, and by (i) we will stay in the category $\mathscr{E}$. Finally, we arrive at the conclusion that there is a domain $F \in \mathscr{E}$ such that the matrix $M_{\mu}$ in (6) belongs to $\mathrm{QPC}\left(F, M_{\mu}(F)\right)$, and that $K\left(F, M_{\mu}(F)\right)\left[M_{\mu}\right] \leqq K_{0}$ for every $\mu$. Furthermore, we can assume that $F$ contains a bidisk $\Delta(0, r)$, and hence

$$
K\left(\Delta(0, r), \Delta^{\mu}(0, r)\right)\left[M_{\mu}\right] \leqq K_{0} \text { for every } \mu,
$$

where $\Delta^{\mu}(0, r)$ stands for the product of two ellipses with axes $\mu r$ and $\mu^{-1} r$. After mapping bianalytically $\Delta(0, r)$ onto $D$ by $\mathscr{S}_{1}$, and $\Delta^{\mu}(0, r)$ onto $D$ by $\mathscr{S}_{2}$, we again arrive at a contradiction, for $\mathscr{S}_{2} \circ M_{\mu} \circ \mathscr{S}_{1}^{-1}$ sends a factor $B$ onto itself with an arbitrarily large constant of quasiconformality. Q.E.D.

\section{REFERENCES}

1. H. Behnke and P. Thullen, Theorie der Funktionen mehrerer komplexer Veränderlichen, Chelsea, New York, 1933.

2. St. Bergman, A class of pseudoconformal and quasi-pseudoconformal mappings, Math. Ann. 136 (1958), 134-138.

3. - A class of quasi-pseudoconformal transformations in the theory of functions of two complex variables, J. Math. Mech. 7 (1958), 937-956.

4. F. W. Gehring, Quasiconformal mappings in space, Bull. Amer. Math. Soc. 69 (1963), 146-164.

5. S. Hitotomatu, On quasiconformal functions of several complex variables, J. Math. Mech. 8 (1959), 77-94.

Courant Institute of Mathematical Sciences, New York University, New York, New YorK 